\title{
Effects of Different Thermal Insulation Methods on Nasopharyngeal Temperature in Patients Undergoing Laparoscopic Hysterectomy: A Prospective Randomized Controlled Trial
}

\section{Guanyu Yang}

First Affiliated Hospital of Zhengzhou University

\section{Zefei Zhu}

First Affiliated Hospital of Zhengzhou University

Hongyu Zheng

First Affiliated Hospital of Zhengzhou University

Shifeng He

First Affiliated Hospital of Zhengzhou University

Wanyue Zhang

First Affiliated Hospital of Zhengzhou University

Zhentao Sun ( $\sim$ gentlesun123@163.com )

First Affiliated Hospital of Zhengzhou University

\section{Research Article}

Keywords: Laparoscopic, Infusion thermometer, Warming blanket, Incubator, Nasopharyngeal temperature

Posted Date: December 3rd, 2020

DOI: https://doi.org/10.21203/rs.3.rs-113986/v1

License: (c) (i) This work is licensed under a Creative Commons Attribution 4.0 International License. Read Full License

Version of Record: A version of this preprint was published at BMC Anesthesiology on April 5th, 2021. See the published version at https://doi.org/10.1186/s12871-021-01324-7. 


\section{Abstract}

Background: The purpose of this study was to compare the thermal insulation effect of incubator and infusion thermometer in laparoscopic hysterectomy.

Methods: 75 patients were enrolled and were assigned randomly to three groups: group A used warming blanket, group B used warming blanket and infusion thermometer, group $\mathrm{C}$ used warming blanket and incubator. The primary outcome was nasopharyngeal temperature at different time points during the operation.

Results: The nasopharyngeal temperature of the infusion heating group was significantly higher than that of the incubator group $60 \mathrm{~min}$ at the beginning of surgery(T3):36.10 \pm 0.20 vs $35.81 \pm 0.20(P \otimes 0.001) \otimes 90 \mathrm{~min}$ at the beginning of surgery(T4):36.35 \pm 0.20 vs $35.85 \pm 0.17(P \otimes 0.001)$, and the incubator group was significantly higher than that of the control group $60 \mathrm{~min}$ at the beginning of surgery(T3):35.81 $\pm 0.20 \mathrm{vs}$ $35.62 \pm 0.18(P \otimes 0.001) ; 90 \mathrm{~min}$ at the beginning of surgery(T4):35.85 \pm 0.17 vs $35.60 \pm 0.17(P \otimes 0.001)$. The wake-up time of the control group was significantly higher than that of the infusion heating group: $23.88 \pm 3.86$ vs $20.56 \pm 3.80(P=0.004)$, and the incubator group: $23.88 \pm 3.86$ vs $21.52 \pm 4.02(P=0.035)$.

Conclusion: Warming blanket $\left(38^{\circ} \mathrm{C}\right)$ combined infusion thermometer $\left(37^{\circ} \mathrm{C}\right)$ provides better perioperative thermal insulation, and in hospitals without infusion thermometer, an incubator can be used as a substitute.

Trial registration: This trial was registered with http://www.chictr.org.cn/index.aspx,ChiCTR2000039162,20 October 2020.

\section{Background}

Perioperative hypothermia is defined as a core temperature below $36^{\circ} \mathrm{C}[1]$, previous studies have demonstrated that the occurrence rate of perioperative hypothermia is between 25 to $90 \%$ [2],perioperative hypothermia may lead to a range of complications, including intraoperative coagulation dysfunction, delayed postoperative recovery, incision infection, etc[3,4].

Laparoscopic surgery has the advantages of less trauma, less bleeding, rapider postoperative recovery, fewer surgical complications, etc $[5,6]$.Compared with open surgeries, the abdominal cavity is relatively closed, but anesthetic factors, persistent $\mathrm{CO}_{2}$ pneumoperitoneum during surgery, and the use of large amounts of irrigating fluid can also lead to hypothermia[7,8].

This study aimed to compare the effects of two different thermal insulation methods in laparoscopic hysterectomy.

\section{Methods}


This prospective, single-blind, randomized, controlled study was approved by the Ethics Committee of the

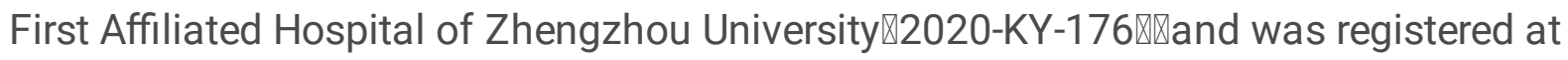
http://www.chictr.org.cn/index.aspx(ChiCTR2000039162) on 20 October 2020. All patients signed the informed consent. Our methodology was executed in accordance with the international guidelines for randomized clinical studies according CONSORT Guidelines.

75 patients scheduled for elective laparoscopic hysterectomy. Inclusion criterion:1) Aged 40-65 years $\llbracket 2$ )

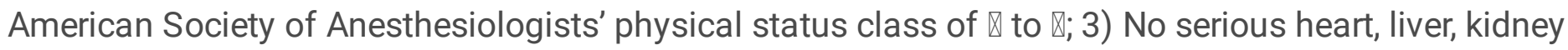
disease;4) No history of severe respiratory or cerebrovascular disease. Exclusion criterion: 1) preoperative anemia; 2) intraoperative blood transfusion; 3) switch to open surgery.75 patients were randomly divided into 3 groups using a random number table: control group(A), infusion heating group(B) or incubator group(C).

\section{Study protocol}

No patients were premedicated and the operating room was kept at $22-24^{\circ} \mathrm{C}$. All three groups use warming blankets and turn them on an hour in advance so that the warming blanket reaches the preset temperature $\left(38^{\circ} \mathrm{C}\right)$. After entering the operating room, patients in the three groups were first injected with $500 \mathrm{ml}$ Ringer's lactate solution and then with $500 \mathrm{ml}$ succinylated gelatin, the infusion speed was controlled at $10 \mathrm{ml} / \mathrm{min}$. In group $A$, the infusion fluid was not treated and the temperature was room temperature; In group B, the infusion fluid was heated by the infusion thermometer, and the target temperature of the infusion thermometer was set at $37^{\circ} \mathrm{C}$; In group $\mathrm{C}$, the infusion fluid was stored in an incubator with the target temperature set at $37^{\circ} \mathrm{C}$.

Nasopharyngeal temperature was monitored after anesthesia induction in all three groups, and vital signs were kept stable during the operation. If the intraoperative nasopharyngeal temperature of the patient is lower than $35^{\circ} \mathrm{C}$, the temperature of the warming blanket will be increased to maintain the nasopharyngeal temperature of the patient above $35^{\circ} \mathrm{C}$.

\section{Outcome measurements}

The primary outcome was nasopharyngeal temperature at $5 \mathrm{~min}$ after anesthesia induction(T1),30 min(T2),60min (T3), and $90 \mathrm{~min}(\mathrm{~T} 4)$ at the beginning of surgery in the three groups. Secondary outcome was wake-up time in three groups.

\section{Statistical analyses}

SPSS was used for all statistical analysis (version 22.0, SPSS Inc, Chicago, IL, USA), measurement data were expressed as mean \pm standard deviation, and analysis of variance was used for comparison between groups, the nasopharyngeal temperature was compared at different time points by repeated measurement ANOVA, Chi-square test was used to compare counting data, and $\mathrm{P}<0.05$ was considered statistically significant. 
The sample size was calculated using GPower (version 3.1.9.2, Franz Faul, Universitat Kiel, Germany), studies have shown that a core temperature difference of $0.5^{\circ} \mathrm{C}$ is clinically significant because $0.5^{\circ} \mathrm{C}$ is the smallest difference associated with hypothermic complications [9]. With the significance level(a) set at 0.05 , power $(1-\beta)$ at 0.9 , then each group needs to include 22 patients, assuming that the rate of withdrawal is $10 \%$, then each group will eventually have 25 patients enrolled.

\section{Results}

A total of 75 patients were included and randomly divided into three groups (Fig. 1). No intraoperative nasopharyngeal temperature was lower than $35^{\circ} \mathrm{C}$.

\section{Primary outcome}

The nasopharyngeal temperature of the infusion heating group was significantly higher than that of the incubator group $60 \mathrm{~min}$ at the beginning of surgery(T3):36.10 \pm 0.20 vs $35.81 \pm 0.20(P \otimes 0.001) \otimes 90 \mathrm{~min}$ at the beginning of surgery(T4):36.35 \pm 0.20 vs $35.85 \pm 0.17(P \otimes 0.001)$, and the incubator group was significantly higher than that of the control group $60 \mathrm{~min}$ at the beginning of surgery(T3):35.81 \pm 0.20 vs $35.62 \pm 0.18(P]$ $0.001) ; 90 \mathrm{~min}$ at the beginning of surgery(T4):35.85 \pm 0.17 vs $35.60 \pm 0.17(P \otimes 0.001)$ (Table 2)(Fig. 3 ).

\section{Secondary outcomes}

The wake-up time of the control group was significantly higher than that of the infusion heating group: $23.88 \pm 3.86$ vs $20.56 \pm 3.80(P=0.004)$, and the incubator group: $23.88 \pm 3.86$ vs $21.52 \pm 4.02(P=0.035)$ (Table

1) (Fig. 2).

Table 1 Descriptive variables of the group A group B and group C

\begin{tabular}{|c|c|c|c|c|c|}
\hline & Group $A \rrbracket n=25 \rrbracket$ & Group B $₫ n=25 \rrbracket$ & Group $C \rrbracket n=25 \rrbracket$ & F value & $P$ value \\
\hline Age $₫$ years $\rrbracket$ & $53.28 \pm 6.91$ & $51.52 \pm 7.6$ & $54.32 \pm 6.30$ & 1.034 & 0.361 \\
\hline BMI $\varangle \mathrm{kg} / \mathrm{m}^{2} \mathrm{a}$ & $23.31 \pm 3.07$ & $23.50 \pm 2.91$ & $23.62 \pm 3.01$ & 0.066 & 0.936 \\
\hline 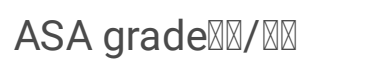 & $13 / 12$ & $16 / 9$ & $12 / 13$ & / & 0.497 \\
\hline Surgery time $\bigotimes \min \rrbracket$ & $108.36 \pm 8.33$ & $111.20 \pm 8.45$ & $109.00 \pm 8.68$ & 0.770 & 0.467 \\
\hline Blood loss $₫ \mathrm{ml} \mathbb{Z}$ & $38.60 \pm 6.70$ & $39.80 \pm 6.69$ & $40.28 \pm 8.84$ & 0.335 & 0.717 \\
\hline 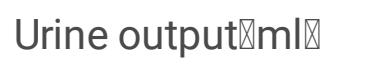 & $338.00 \pm 96.05$ & $340.00 \pm 76.38$ & $358.00 \pm 89.77$ & 0.394 & 0.676 \\
\hline Wake-up time $₫ \min \rrbracket$ & $23.88 \pm 3.86$ & $20.56 \pm 3.80$ & $21.52 \pm 4.02$ & 4.816 & 0.011 \\
\hline
\end{tabular}

Values are presented as mean (standard deviation) or counts

ASA American society of anesthesiologists; $B M /$ Body Mass Index 
Table 2 Nasopharyngeal temperature at different points in three groups

\begin{tabular}{|llllll|}
\hline Group & $\mathrm{n}$ & $\mathrm{T} 1$ & T2 & T3 & T4 \\
\hline A & 25 & $36.40 \pm 0.19$ & $35.88 \pm 0.21^{\mathrm{t}}$ & $35.62 \pm 0.18^{\mathrm{t}}$ & $35.60 \pm 0.17^{\mathrm{t}}$ \\
\hline B & 25 & $36.37 \pm 0.24$ & $35.92 \pm 0.24^{\mathrm{t}}$ & $36.10 \pm 0.20^{\text {*t }}$ & $36.35 \pm 0.20^{*}$ \\
\hline C & 25 & $36.35 \pm 0.21$ & $35.88 \pm 0.18^{\mathrm{t}}$ & $35.81 \pm 0.20^{\text {*\#t }}$ & $35.85 \pm 0.17^{\text {*\#t }}$ \\
\hline
\end{tabular}

${ }^{*} P \bowtie 0.05$ compared to the same time of Group A

${ }^{\#} P \otimes 0.05$ compared to the same time of Group B

${ }^{\mathrm{t}} P \otimes 0.05$ compared to $\mathrm{T} 1$

Values are presented as mean \pm standard deviation

$5 \mathrm{~min}$ after anesthesia induction( $\mathrm{T} 1), 30 \mathrm{~min}$ at the beginning of surgery(T2), $60 \mathrm{~min}$ at the beginning of surgery(T3), $90 \mathrm{~min}$ at the beginning of surgery(T4)

\section{Discussion}

The results of this study showed that nasopharyngeal temperature decreased significantly in the three groups from T1 to T2. On the one hand, general anesthesia leads to peripheral vasodilation, which inhibits the function of blood vessels to regulate body temperature through contraction; meanwhile, general anesthesia also inhibited the body's central thermoregulation, and anesthetic drugs also decreased the metabolic rate[10,11]. On the other hand, the operating room temperature is relatively low, and a lot of heat will be absorbed by preoperative disinfection and intraoperative infusion, as well as heat will be taken away by continuous $\mathrm{CO}_{2}$ infusion during the operation $[8,12]$.

The nasopharyngeal temperature of the T2 to T3 control group and the incubator group continued to decrease, and the decrease degree of the incubator group was less than that of the control group. The nasopharyngeal temperature of the infusion heating group began to increase, indicating that the use of a warming blanket alone $\left(38^{\circ} \mathrm{C}\right)$ could not maintain the patient's body temperature, and might require a higher temperature. The infusion fluid in the incubator group was not continuously heated, and the temperature gradually decreased over time. Only the infusion heating group made the patient's body temperature rise by warming blanket and infusion thermometer.

The nasopharyngeal temperature of the T3 to T4 control group began to stabilize, and the nasopharyngeal temperature of the incubator group began to rise, but was lower than $\mathrm{T} 1$. The nasopharyngeal temperature of the infusion heating group continued to rise, with no significant difference from $\mathrm{T} 1$, indicating that the temperature of the patients could be maintained to the 
preoperative level when the warming blanket $\left(38^{\circ} \mathrm{C}\right)$ combined infusion thermometer $\left(37^{\circ} \mathrm{C}\right)$ was used for $90 \mathrm{~min}$.

The wake-up time of the control group was significantly higher than that of the infusion heating group and the incubator group, mainly because of the low temperature status, which reduced the capacity of the liver to uptake drugs and the capacity of the kidney to excrete drugs, which affected the metabolism of anesthetic drugs in the body and led to prolonged wake-up time $[13,14]$.

As one of the important vital signs, body temperature has attracted more and more attention in the perioperative period in recent years. However, the protection of body temperature during perioperative period is not good. On the one hand, this is due to the insufficient attention paid by medical staff to body temperature protection; on the other hand, there is no protective device for body temperature in hospitals, such as infusion thermometer $[15,16]$. The incubator is used as a necessary equipment for hospitals, usually to preserve the irrigating fluid used during the operation. This study makes full use of the equipment to preserve the fluid injected during the operation to achieve the effect of fluid heating. Although the patient has hypothermia during the operation, the temperature drop is slight and the wakeup time was not prolonged. Therefore, for hospitals without better insulation device, this method is easy to use, low cost and worth popularizing.

The observation time in this study was limited to $90 \mathrm{~min}$, for longer duration of surgery, it is not clear whether the temperature of the warming blanket and infusion thermometer should be adjusted, and further research is still needed.

\section{Conclusions}

Warming blanket $\left(38^{\circ} \mathrm{C}\right)$ combined infusion thermometer $\left(37^{\circ} \mathrm{C}\right)$ provides better perioperative thermal insulation, and in hospitals without infusion thermometer, an incubator can be used as a substitute.

\section{Abbreviations}

BMI: Body Mass Index; ASA: American Society of Anesthesiologists

\section{Declarations}

\section{Acknowledgements}

This research was supported by the department of gynaecology of the First Affiliated Hospital of Zhengzhou University.

\section{Author's contributions}

Study design: GY, ZZ, ZS. Study conduct: GY, HZ, SH. Data analysis: GY, ZZ, WZ, ZS. Writing paper: GY, ZZ, HZ, SH, WZ, ZS. All authors read and approved the final manuscript version. 


\section{Funding}

This study was supported by the Henan Provincial Science and Technology Research Project (2018010006).

\section{Availability of data and materials}

The raw data of this study are available from the corresponding author on reasonable request.

\section{Ethics approval and consent to participate}

This study was approved by the Ethics Committee of the First Affiliated Hospital of Zhengzhou University on May 31, 2020ه2020-KY-176冈. All patients signed the informed consent.

\section{Consent for publication}

Not applicable.

\section{Competing interests}

None.

\section{References}

1. John M, Crook D, Dasari K, Eljelani F, El-Haboby A, Harper C M. Comparison of resistive heating and forced-air warming to prevent inadvertent perioperative hypothermia. $\mathrm{Br} \mathrm{J}$ Anaesth, 2016, 116: 24954.

2. Torossian Alexander, Bräuer Anselm, Höcker Jan, Bein Berthold, Wulf Hinnerk, Horn Ernst-Peter. Preventing inadvertent perioperative hypothermia. Dtsch Arztebl Int, 2015, 112: 166-72.

3. Rajagopalan Suman, Mascha Edward, Na Jie, Sessler Daniel I. The effects of mild perioperative hypothermia on blood loss and transfusion requirement. Anesthesiology, 2008, 108: 71-7.

4. Melling AC, Ali B, Scott EM, Leaper DJ. Effects of preoperative warming on the incidence of wound infection after clean surgery: a randomised controlled trial. Lancet, 2001; 358: 876-80

5. Li Min, Cao Baoqiang, Gong Renhua, Sun Dengqun, Zhang Peisong, Jiang Xudong, Sheng Yanfei. Randomized trial of umbilical incisional hernia in high-risk patients: extraction of gallbladder through subxiphoid port vs. umbilical port after laparoscopic cholecystectomy . Wideochir Inne Tech Maloinwazyjne, 2018, 13: 342-9.

6. Wang Tao, Liu Hui, Sun Jian Hong, Wang Lin, Zhang Jian You. Efficacy of intravenous lidocaine in improving post-operative nausea, vomiting and early recovery after laparoscopic gynaecological surgery . Exp Ther Med, 2019, 17: 4723-9.

7. Balki Indranil, Khan James S, Staibano Phillip, Duceppe Emmanuelle, Bessissow Amal, Sloan Erin N, Morley Erin E, Thompson Alexandra N, Devereaux Breagh, Rojas Constanza, Rojas Camila, Siddiqui 
Naveed, Sessler Daniel I, Devereaux P J. Effect of Perioperative Active Body Surface Warming Systems on Analgesic and Clinical Outcomes: A Systematic Review and Meta-analysis of Randomized Controlled Trials. Anesth Analg, 2020, 131: 1430-43.

8. Noll Eric, Diemunsch Sophie, Pottecher Julien, Rameaux Jean-Pierre, Diana Michele, Sauleau Eric, Ruetzler Kurt, Diemunsch Pierre. Prevention of laparoscopic surgery induced hypothermia with warmed humidified insufflation: Is the experimental combination of a warming blanket synergistic? PLoS One, 2018, 13: e0199369.

9. Winkler M, Akça O, Birkenberg B, Hetz H, Scheck T, Arkiliç C F, Kabon B, Marker E, Grübl A, Czepan R, Greher M, Goll V, Gottsauner-Wolf F, Kurz A, Sessler D I. Aggressive warming reduces blood loss during hip arthroplasty. Anesth Analg. 2000;91(4):978-84

10. Schacham Yehoshua N, Cohen Barak, Bajracharya Gausan R, Walters Michael, Zimmerman Nicole, Mao Guangmei, Tanios Marianne A, Sessler Daniel I. Mild Perioperative Hypothermia and Myocardial Injury: A Retrospective Cohort Analysis. Anesth Analg, 2018, 127: 1335-41.

11. Rein E B, Filtvedt M, Walløe L, Raeder J C. Hypothermia during laparotomy can be prevented by locally applied warm water and pulsating negative pressure. $\mathrm{Br}$ J Anaesth, 2007, 98: 331-6.

12. Andrzejowski J, Hoyle J, Eapen G, Turnbull D. Effect of prewarming on post-induction core temperature and the incidence of inadvertent perioperative hypothermia in patients undergoing general anaesthesia. Br J Anaesth, 2008, 101: 627-31.

13. Triffterer Lydia, Marhofer Peter, Sulyok Irene, Keplinger Maya, Mair Stefan, Steinberger Markus, Klug Wolfgang, Kimberger Oliver. Forced-Air Warming During Pediatric Surgery: A Randomized Comparison of a Compressible with a Noncompressible Warming System. Anesth Analg, 2016, 122: 219-25.

14. Lenhardt R, Marker E, Goll V, Tschernich H, Kurz A, Sessler D I, Narzt E, Lackner F. Mild intraoperative hypothermia prolongs postanesthetic recovery. Anesthesiology, 1997, 87: 1318-23.

15. Torossian A, TEMMP (Thermoregulation in Europe Monitoring and Managing Patient Temperature) Study Group. Survey on intraoperative temperature management in Europe. Eur J Anaesthesiol, 2007, 24: 668-75.

16. Boet Sylvain, Patey Andrea M, Baron Justine S, Mohamed Karim, Pigford Ashlee-Ann E, Bryson Gregory L, Brehaut Jamie C, Grimshaw Jeremy M. Factors that influence effective perioperative temperature management by anesthesiologists: a qualitative study using the Theoretical Domains Framework. Can J Anaesth, 2017, 64: 581-96.

\section{Figures}




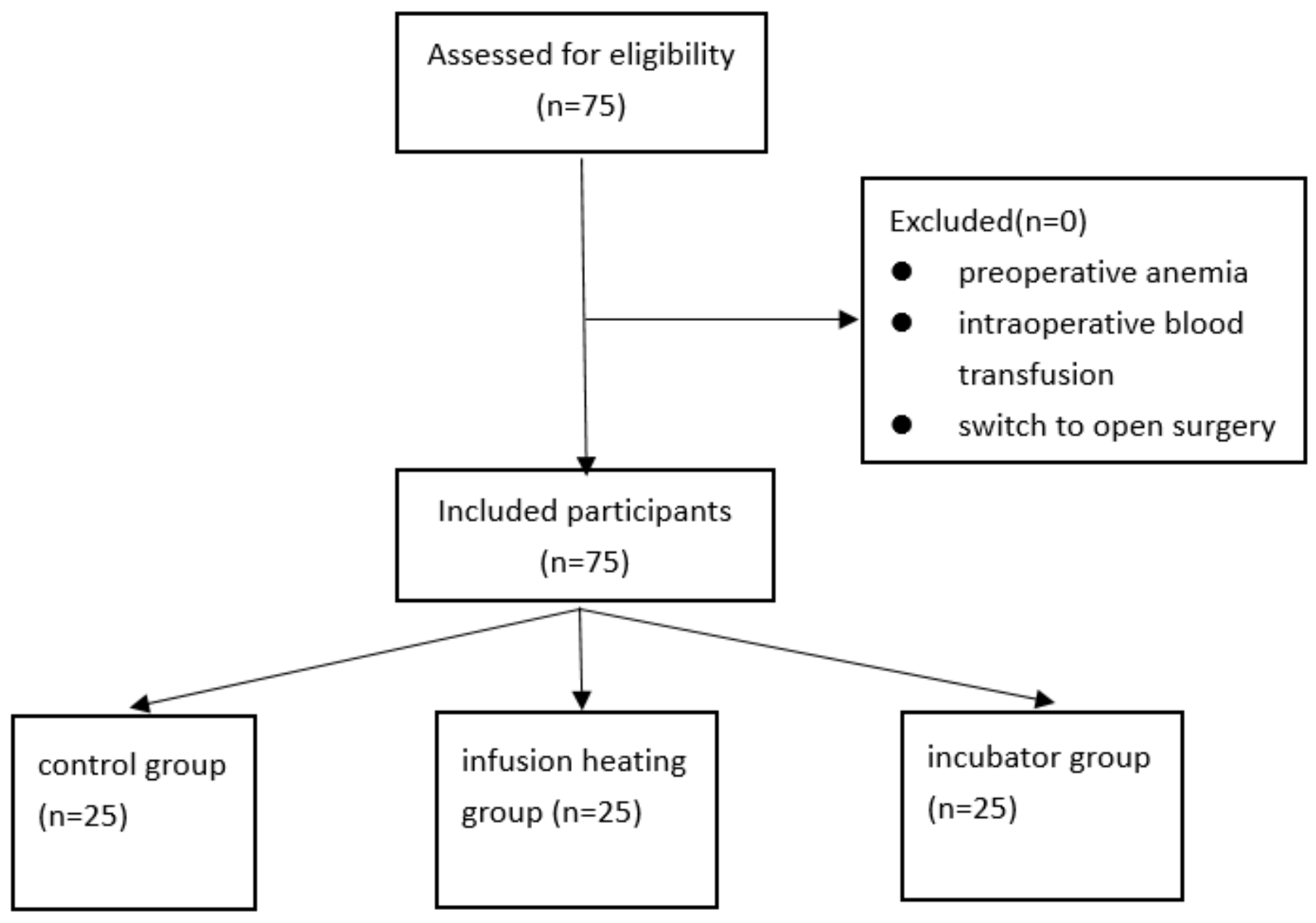

Figure 1

Participant flowchart 


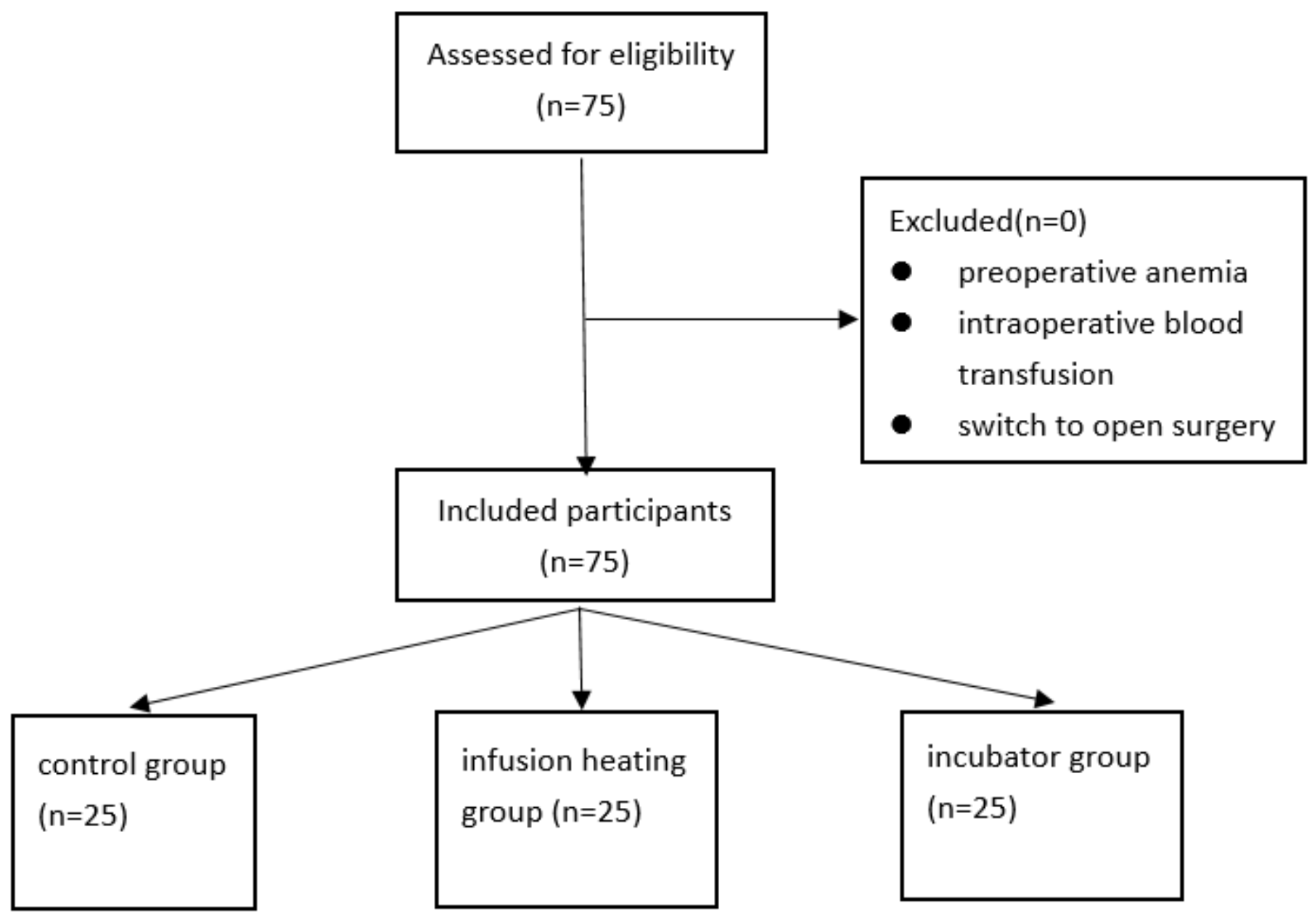

Figure 1

Participant flowchart

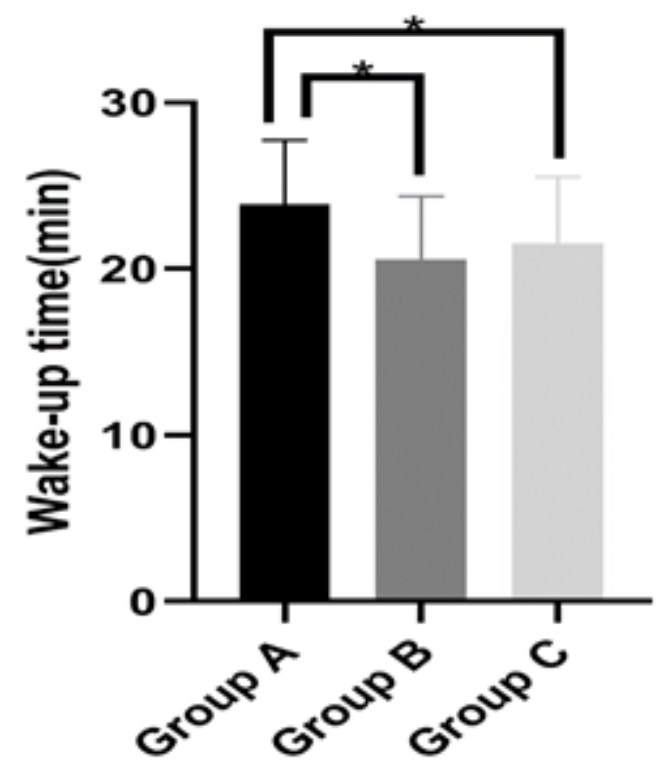

Figure 2 
Wake-up time in three groups; ${ }^{*} \mathbb{P} \otimes 0.05$

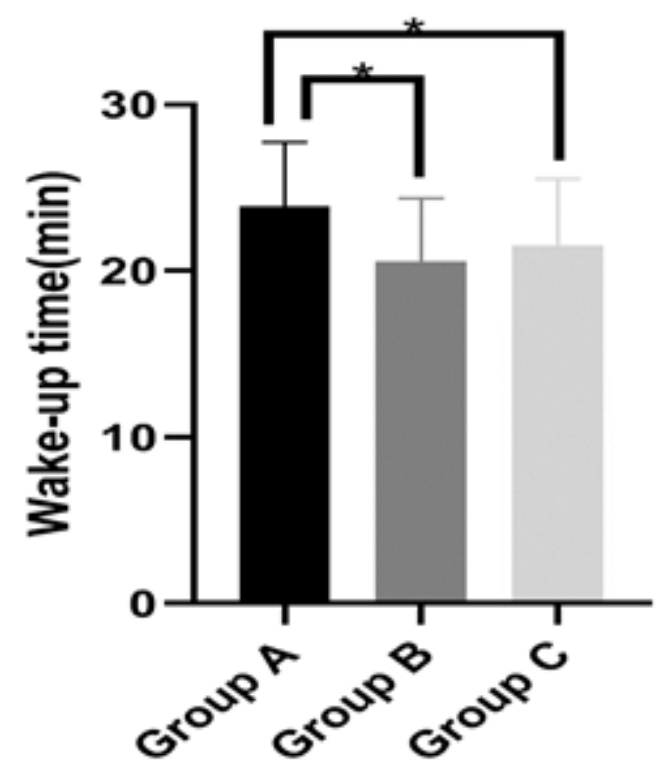

Figure 2

Wake-up time in three groups; $\mathrm{P} \otimes 0.05$

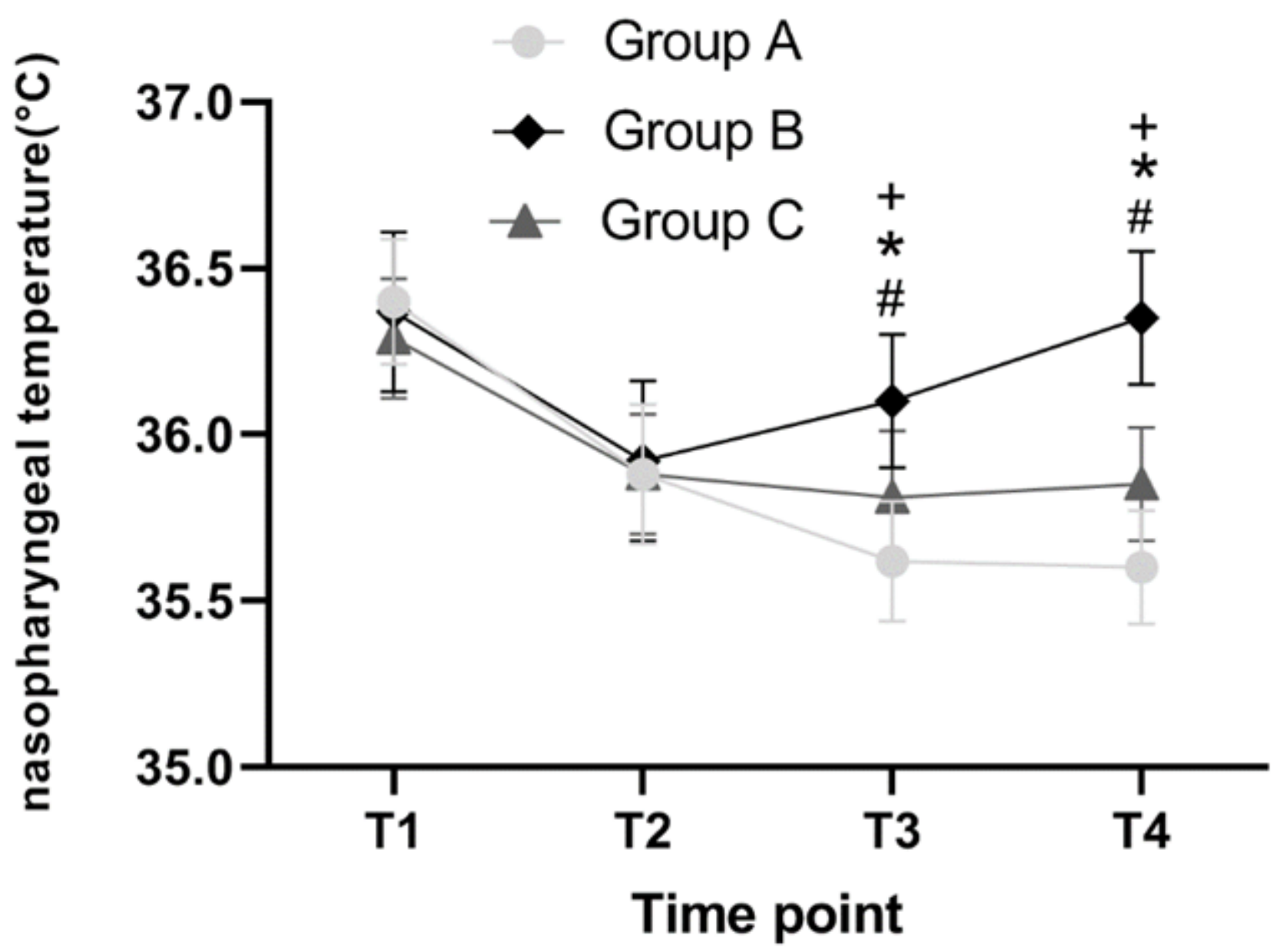


Figure 3

Nasopharyngeal temperature at different points in three groups. Time point, $5 \mathrm{~min}$ after anesthesia induction(T1), 30min at the beginning of surgery(T2), $60 \mathrm{~min}$ at the beginning of surgery(T3), $90 \mathrm{~min}$ at the beginning of surgery(T4); +statistical significance between the group A and group B; *statistical significance between the group $A$ and group $C$; \#statistical significance between the group $B$ and group $C$

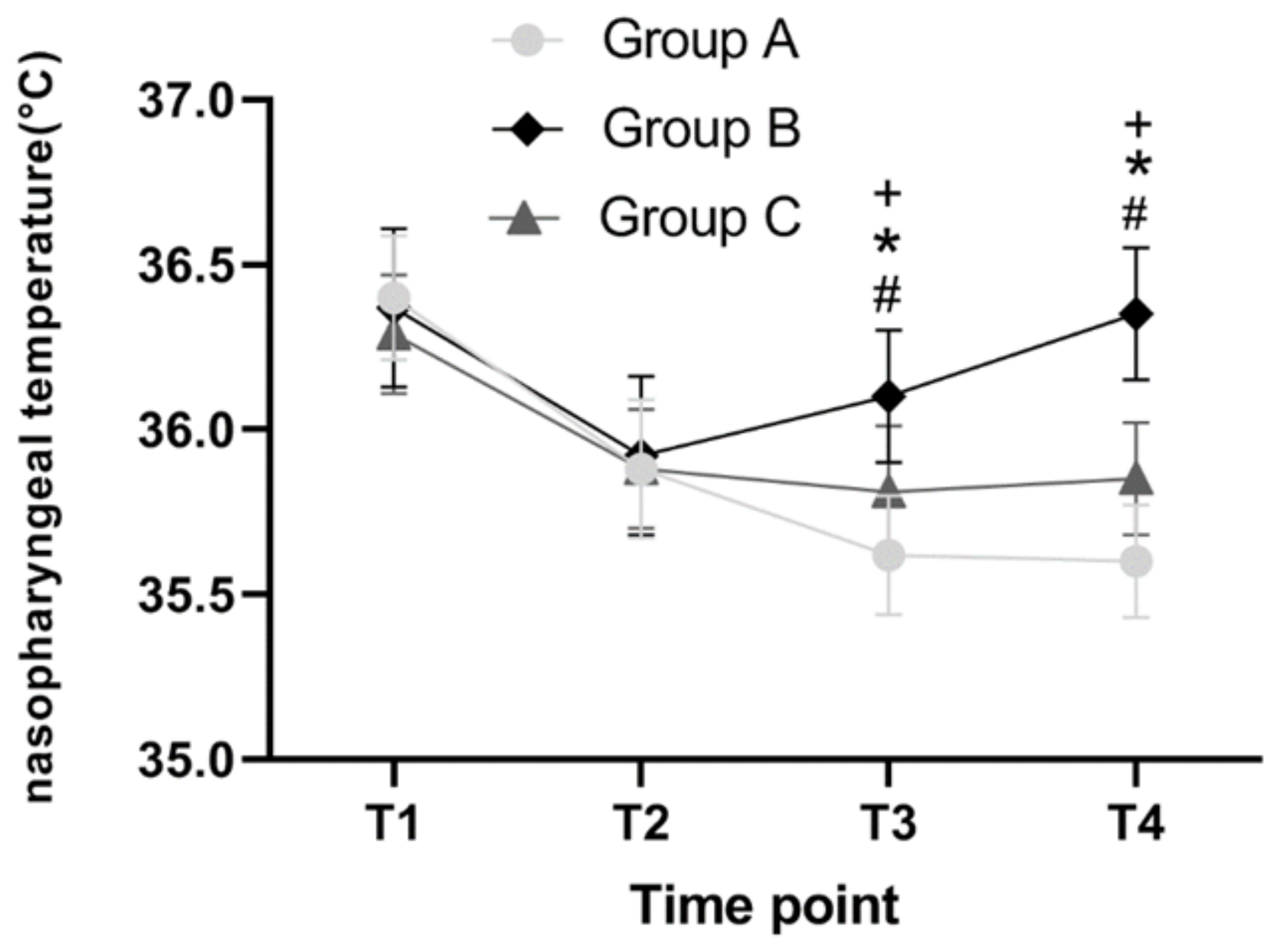

Figure 3

Nasopharyngeal temperature at different points in three groups. Time point, $5 \mathrm{~min}$ after anesthesia induction(T1), 30min at the beginning of surgery(T2), $60 \mathrm{~min}$ at the beginning of surgery(T3), $90 \mathrm{~min}$ at the beginning of surgery(T4); +statistical significance between the group A and group B; *statistical significance between the group A and group C; \#statistical significance between the group $B$ and group $C$ 